\title{
Marginally outer trapped surfaces in higher dimensions *
}

\author{
Tim-Torben Paetz ${ }^{\dagger}$ and Walter Simon ${ }^{\ddagger}$ \\ Gravitationsphysik, Fakultät für Physik, Universität Wien \\ Boltzmanngasse 5, A-1090 Wien, Austria
}

\begin{abstract}
We review the basic setup of Kaluza-Klein theory, namely a 5-dimensional vacuum with a cyclic isometry, which corresponds to Einstein-Maxwell-dilaton theory in 4-dimensional spacetime. We first recall the behaviour of Killing horizons under bundle lift and projection. We then show that the property of compact surfaces of being (stably) marginally trapped is preserved under lift and projection provided the appropriate ("Pauli-") conformal scaling is used for the spacetime metric. We also discuss and compare recently proven area inequalities for stable axially symmetric 2-dimensional and 3-dimensional marginally outer trapped surfaces.
\end{abstract}

\section{Introduction}

The basic setup of Kaluza-Klein (KK) theory consists of a $4+\mathrm{n}$ dimensional manifold with n Killing vectors, which can be regarded as a principal fibre bundle over a $4 \mathrm{~d}$ base (see e.g. [1, 2]). In 5 dimensions in particular, stationary black holes have been studied extensively and revealed much richer structures than in $4 \mathrm{~d}$ (cf e.g. [3]-[8]). There is, however, a more subtle motivation for studying black holes in $(4+\mathrm{n})$ dimensions: Suitable adaptions of Hawking's rigidity theorem assert the existence of $n+1$ spacelike isometries on generic (non-static) stationary event horizons [9], and under suitable technical assumptions, such Killing fields can be extended off the horizon. From this point of view, the higher dimensional black hole is the "fundamental" object which by itself is capable of "generating $4 \mathrm{~d}$ spacetime" with matter fields, via n isometries which serve as KK-fibres [6]. In addition, the horizon "generates" axial symmetry as usual.

In more realistic KK theories the $\mathrm{n}$ fibre-isometries are replaced by periodicity conditions on all fields involved (cf e.g. [1]). This raises the question whether such structures could arise "automatically" as well, namely from a non-stationary but suitably structured horizon, as a consequence of a hypothetical generalized rigidity theorem. While a concrete implementation

*Preprint UWThPh-2013-7

†tim-torben.paetz@univie.ac.at

†walter.simon@univie.ac.at 
of this speculation would lead beyond the scope of the present work, the basic idea does give a motivation for studying general gravitational collapse in higher dimensions, as initiated below.

The key concepts in the study of gravitational collapse are (outer) trapped surfaces and marginally outer trapped surfaces (MOTS). The latter are defined as compact codimension 2surfaces on which the orthogonally outgoing family of null geodesics has vanishing expansion (cf e.g. [10]). In applications, "stable" and "strictly stable" MOTS play a distinguished role and have been studied extensively [10]-[20]. Here stability is a mild restriction which roughly speaking requires the existence of a codimension 1-neighbourhood in a selected normal direction, which can be foliated by trapped surfaces inside and untrapped surfaces outside. On the other hand, it has been shown that the (global) boundary of the trapped region in a spacelike hypersurface is formed by a smooth, stable MOTS [13, 14, 15]. Originally, the stability condition came up in connection with the topology theorems for MOTS [16, 17, 18]. More recently, however, the crucial role of stability in the time evolution of MOTS in a foliated spacetime was clarified, as stability implies smooth evolution while instability signals "jumps" [19. Moreover, stability is significant for the singularity theorems, as the original requirement of the existence of a trapped surface (both null expansions converging) can in essence be replaced by the requirement of existence of a stable MOTS [20]. All these results apply in principle in higher dimensions, (at least till 7 ), and motivate our interest in the stability condition in particular.

A further motivation comes from the area inequalities for stable MOTS [21, 22, 23]. Such inequalities have been found in particular for axially symmetric, stable 2d MOTS in 4d EinsteinMaxwell by Gabach Clement, Jaramillo and Reiris [24, 25] and in 4d Einstein-Maxwell dilaton (EMD) theory by Yazadjiev [26]. Other inequalities have been obtained for stable 3d MOTS with various topologies and corresponding symmetries in $5 \mathrm{~d}$ spacetimes by Hollands [27]. Since a $5 \mathrm{~d}$ vacuum with a KK symmetry is equivalent to EMD theory in $4 \mathrm{~d}$, the results of these papers can be compared provided the concepts of stable 2d MOTS and 3d MOTS can be matched. These are the main goals of the present paper.

As we want to illustrate the basic concepts rather than investigating a physically realistic theory, we restrict ourselves to $5 \mathrm{~d}$ vacuum spacetimes and their dimensional reduction along an isometry. We first (in Sect. 2) review Geroch's projection formalism [28] and continue in Sect. 3.1 with recalling the known behaviour of Killing horizons and their generators under such a reduction [6, 9]. In Sect. 3.2 we show that 3d MOTS do project down to $2 \mathrm{~d}$ ones provided an appropriate conformal scaling (which may be ascribed to Pauli [29]) is used for the spacetime metric. The scaling is irrelevant only in a degenerate case in which the norm of the KK Killing field is constant along the outgoing null direction. Fortunately, the Pauliscaling is the same which is compulsory for matching the variational formulations in different dimensions anyway [1, 30]. Next (in Sect. 3.3) we relate the stability definitions for 3d and 2d MOTS, which is straightforward in principle but involves some subtleties. In Sect. 4 we finally analyze and relate the area inequalities. This requires a careful discussion of the parameters and of the non-trivial topologies which arise in the presence of a magnetic monopole. 


\section{Kaluza-Klein a la Geroch}

We consider a smooth 5-dimensional Ricci-flat manifold $\left({ }^{5} \mathcal{M},{ }^{5} g_{A B}\right)$ of signature $(-++++)$, where capital latin indices run from 0 to 4 . We assume that $\left({ }^{5} \mathcal{M},{ }^{5} g_{A B}\right)$ is a principal fibre bundle with spacelike $U(1)$ isometry $\mathcal{C}$ as fibre and smooth base manifold $\mathcal{M}$ (cf. [2]). This entails that $\mathcal{C}$ has no fixed points; the corresponding Killing field on ${ }^{5} \mathcal{M}$ is denoted by $c^{A}$, i.e. the corresponding Lie derivative satisfies $\mathcal{L}_{c}{ }^{5} g_{A B}=0$. The squared norm of $c^{A}$ is denoted by $V^{2}=c^{A} c_{A}>0$. Subsets of ${ }^{5} \mathcal{M}$ are distinguished from subsets of $\mathcal{M}$ by the superscript ${ }^{5}$ (irrespective of their dimension). Before introducing a coordinate system in Equ. (5), tensorial objects carry (Penrose's) "abstract indices " to indicate their nature; in particular, tensors on ${ }^{5} \mathcal{M}$ carry capital indices while those on $\mathcal{M}$ carry lower case indices ranging from 0 to 3 . A set $\mathcal{E} \subset{ }^{5} \mathcal{M}$ is called invariant if $\mathcal{C}(\mathcal{E})=\mathcal{E}$.

Below we introduce a terminology which is some sort of amalgamation between standard fibre bundle language and Geroch's projection formalism [28]; However, for sets the terminology "lift" and "projection" used below is not standard, while for vectors we drop the word "horizontal" from the standard term "horizontal lift"; see the remark at the end of this section.

The lift $\mathcal{C}^{\uparrow}$ of a set $\mathcal{D}$ :

$\mathcal{C}^{\uparrow}: \mathcal{M} \rightarrow{ }^{5} \mathcal{M}$ assigns to a subset $\mathcal{D} \subset \mathcal{M}$ the set of points $\mathcal{E} \subset{ }^{5} \mathcal{M}$ on the orbits through $\mathcal{D}$.

\section{The projection $\mathcal{C}^{\downarrow}$ of an invariant set $\mathcal{E}$ :}

$\mathcal{C}^{\downarrow}:{ }^{5} \mathcal{M} \rightarrow \mathcal{M}$ assigns to an invariant subset $\mathcal{E} \subset{ }^{5} \mathcal{M}$ the set of orbits $\mathcal{E} / \mathcal{C}$.

These maps induce maps between invariant tensor fields $w^{A \ldots M}{ }_{N \ldots Z}$ on ${ }^{5} \mathcal{M}$, defined by

$$
\mathcal{L}_{c} w^{A \ldots M}{ }_{N \ldots Z}=0, \quad w^{A \ldots M}{ }_{N \ldots Z} c_{A}=0 \quad \ldots \quad w^{A \ldots M}{ }_{N \ldots Z} c^{Z}=0
$$

and tensor fields on $\mathcal{M}$. The maps displayed in Fig. 1 and defined below are of course nothing but "pullbacks" and "pushforwards".

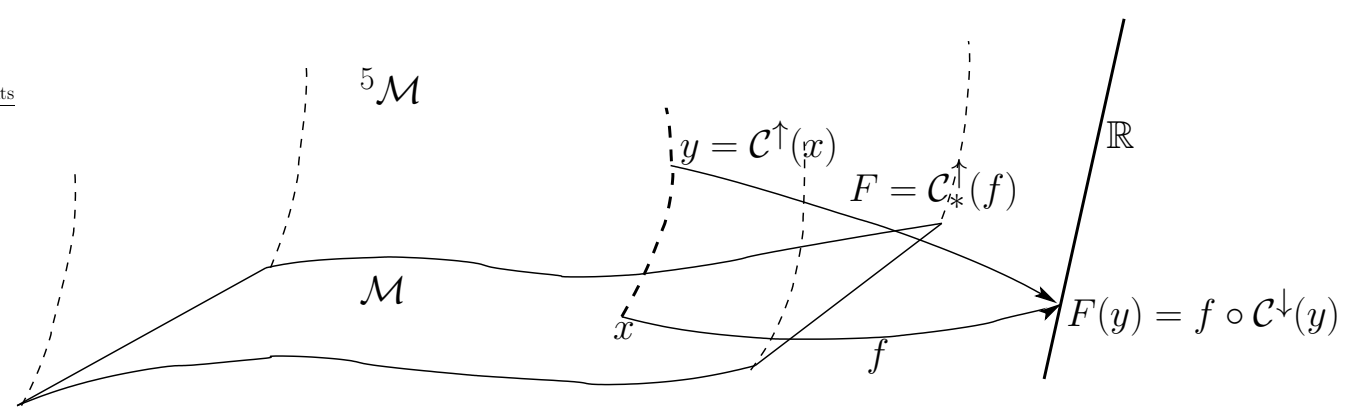

Figure 1: Lifts and projections of functions

Lift $\mathcal{C}_{*}^{\uparrow}$ of a function $f: \mathcal{M} \rightarrow \mathbb{R} ; f \in \mathcal{F}(\mathcal{M})$ (the module of functions) to an invariant function $F:{ }^{5} \mathcal{M} \rightarrow \mathbb{R} ; F \in \mathcal{F}_{-}\left({ }^{5} \mathcal{M}\right)$ (the module of invariant functions): $\mathcal{C}_{*}^{\uparrow}: \mathcal{F}(\mathcal{M}) \rightarrow \mathcal{F}_{-}\left({ }^{5} \mathcal{M}\right)$ is defined as $F=\mathcal{C}_{*}^{\uparrow}(f)=f \circ \mathcal{C}^{\downarrow}$. 
Projection of an invariant function $F \in \mathcal{F}_{-}\left({ }^{5} \mathcal{M}\right)$ :

$\mathcal{C}_{*}^{\downarrow}: \mathcal{F}_{-}\left({ }^{5} \mathcal{M}\right) \rightarrow \mathcal{F}(\mathcal{M})$ is defined as $f=\mathcal{C}_{*}^{\downarrow}(F)=F \circ \mathcal{C}^{\uparrow}$.

The maps introduced above can now be extended straightforwardly to vector and tensor fields by considering gradients of functions etc.. For definitions cf Appendix A of [28] - here we just fix notations:

Lift $\mathcal{C}_{*}^{\uparrow}$ of a vector field $w^{i} \in T(\mathcal{M})$ to an invariant vector field $z^{A} \in T_{-}\left({ }^{5} \mathcal{M}\right)$ :

(the horizontal subspace of $T\left({ }^{5} \mathcal{M}\right)$ ).

$\mathcal{C}_{*}^{\uparrow}: T(\mathcal{M}) \rightarrow T_{-}\left({ }^{5} \mathcal{M}\right)$ denoted by $\mathcal{C}_{*}^{\uparrow}\left(w^{i}\right)=z^{A}$.

Projection of an invariant vector field $z^{A}$ :

$\mathcal{C}_{*}^{\downarrow}: T_{-}\left({ }^{5} \mathcal{M}\right) \rightarrow T(\mathcal{M})$ denoted by $\mathcal{C}_{*}^{\downarrow}\left(z^{A}\right)=w^{i}$.

From the construction it is clear that $\mathcal{C}^{\uparrow}$ and $\mathcal{C}^{\downarrow}$ are not isomorphisms, while $\mathcal{C}_{*}^{\uparrow}$ and $\mathcal{C}_{*}^{\downarrow}$ are [28], which is indicated by the asterisks. In what follows $\mathcal{C}_{*}^{\downarrow}$ will only be applied to invariant objects. $\mathcal{C}_{*}^{\uparrow}$ and $\mathcal{C}_{*}^{\downarrow}$ are homomorphisms in the sense that they cut through tensor products. In Lemma 1 below, the action of these mappings on covariant and contravariant vector fields will be displayed explicitly in the coordinate system (5) adapted to the isometry.

The projection maps introduced above should not be confused with a particular tensor field called "the projector" defined by

$$
P_{C}^{A}=\delta_{C}^{A}-V^{-2} c^{A} c_{C},
$$

which can in fact be applied to any tensor field in $\otimes T\left({ }^{5} \mathcal{M}\right)$. In particular, applied to ${ }^{5} g_{A B}$ it gives the invariant metric

$$
\widetilde{g}_{C D}=P_{C}^{A} P_{D}^{B}{ }^{5} g_{A B}
$$

which effectively lives on $\mathcal{M}$; in our notation this reads $\mathcal{C}^{\downarrow}\left(\widetilde{g}_{A B}\right)=\widetilde{g}_{i j}$. We also recall the volume forms $\widetilde{\mu}=* 1$ on $\mathcal{M}$ and ${ }^{5} \mu={ }^{5} * 1$ on ${ }^{5} \mathcal{M}$ where $*$ and ${ }^{5} *$ are the Hodge duals, and the antisymmetric symbols $\widetilde{\epsilon}_{i j k l}$ with $\epsilon_{0123}=\sqrt{\operatorname{det} \widetilde{g}}$ and $\epsilon_{A B C D E}$ with $\epsilon_{01234}=\sqrt{\operatorname{det}{ }^{5} g}$. These objects are related via $\mathcal{C}_{*}^{\uparrow}\left(\widetilde{\epsilon}_{i j k l}\right)=V^{-1} \epsilon_{A B C D E} c^{E}$.

The Levi-Civita conection w.r.t. ${ }^{5} g_{A B}$ is denoted by ${ }^{5} \nabla_{A}$. For an invariant vector field $w^{A}$ another covariant derivative $\widetilde{\nabla}_{A}$ can be defined by [28]

$$
\widetilde{\nabla}_{C} w_{D}=P_{C}^{A} P_{D}^{B}{ }^{5} \nabla_{A} w_{B}
$$

The derivative $\widetilde{\nabla}_{A}$ is metric with respect to (3), torsion free and $\widetilde{\nabla}_{C} w_{D}$ is invariant in the sense of (11). Hence it is identical with the Levi-Civita connection with respect to $\widetilde{g}_{i j}$ on $\mathcal{M}$, and we can write $\mathcal{C}_{*}^{\downarrow}\left(\widetilde{\nabla}_{A} w_{B}\right)=\widetilde{\nabla}_{i} w_{j}$.

Below we will almost exclusively use the conformally rescaled metric $g_{i j}=v \widetilde{g}_{i j}=\mathcal{C}_{*}^{\downarrow}\left(V \widetilde{g}_{A B}\right)$ on $\mathcal{M}$; in fact this extraction of $v=\mathcal{C}_{*}^{\downarrow}(V)$ will be crucial. The covariant derivative w.r.t $g_{i j}$ is denoted by $\nabla_{i}$.

A parametrisation of ${ }^{5} g_{A B}$ which will be useful in what follows is

$$
d s^{2}={ }^{5} g_{A B} d x^{A} d x^{B}=V^{2}\left(d x^{4}+2 A_{i} d x^{i}\right)^{2}+V^{-1} g_{i j} d x^{i} d x^{j},
$$


where $V, A_{i}$ and $g_{i j}$ depend on $x^{i}$ only. Such coordinates exist and will be used below on neighbourhoods $\mathcal{N}$ of compact 2-surfaces $\mathcal{S} \subset \mathcal{M}$ and of the corresponding lift ${ }^{5} \mathcal{N}=\mathcal{C}^{\uparrow}(\mathcal{N}) \supset$ ${ }^{5} \mathcal{S}$. From now onwards, all "abstract" indices materialize as coordinate indices w.r.t. (5). The coordinate $x^{4}$ is periodic with period $Z$, i.e. $x^{4}$ is identified with $x^{4}+Z$.

Instead of $V$ a more common terminology is either $V=e^{2 \varphi / \sqrt{3}}[5]$ or $V=e^{-2 \varphi / \sqrt{3}}$ [4]. Our definition of $A_{i}$ follows practice in relativity [4, 5, 6] but differs from practice in gauge theory at least by a factor of 2 [2], if not by charge-like constants as well.

The Lagrangian density on $\left({ }^{5} \mathcal{M},{ }^{5} g_{A B}\right)$ can be decomposed as [4, 5]

$$
£=\frac{\sqrt{{ }^{5} g}}{16 \pi Z}{ }^{5} R=\frac{\sqrt{g}}{16 \pi}\left[R-\frac{3}{2} v^{-2} \nabla_{i} v \nabla^{i} v-v^{3} F_{i j} F^{i j}\right],
$$

where

$$
F_{i j}=\mathcal{C}_{*}^{\downarrow}\left({ }^{5} \nabla_{[A}\left(V^{-2} c_{B]}\right)\right)=2 \nabla_{[i} A_{j]} ;
$$

note that the argument of $\mathcal{C}_{*}^{\downarrow}$ is indeed an invariant object.

This Lagrangian determines EMD theory on $\left(\mathcal{M}, g_{i j}\right)$. As well known, the metric variable $A_{i}$ in (5), which is the dynamic variable in (6), can be gauge transformed via $A_{i} \rightarrow A_{i}+\nabla_{i} \Lambda$ for some function $\Lambda$, and this corresponds to the coordinate transformation $x^{4} \rightarrow x^{4}+2 \Lambda$ in (5).

We now give the action of $\mathcal{C}_{*}^{\uparrow}$ explicitly in the coordinates (5).

Lemma 1. Let $y_{i}$ and $w^{i}$ be co- and contravariant vector fields on $\left(\mathcal{M}, g_{i j}\right)$. On $\left({ }^{5} \mathcal{M}, g_{A B}\right)$ and in adapted coordinates (15), we define $y_{A}=\left(y_{i}, 0\right), w^{A}=\left(w^{i}, 0\right)$ and $\alpha=2 w^{i} A_{i}$. Then the lifts $\mathcal{C}_{*}^{\uparrow}\left(y_{i}\right)$ and $\mathcal{C}_{*}^{\uparrow}\left(w^{i}\right)$ are given in these coordinates by

$$
\begin{aligned}
\mathcal{C}_{*}^{\uparrow}\left(y_{i}\right) & =y_{A}, \\
\mathcal{C}_{*}^{\uparrow}\left(w^{i}\right) & =z^{A}:=w^{A}-\mathcal{C}_{*}^{\uparrow}(\alpha) c^{A} .
\end{aligned}
$$

Proof. It is easy to see that the vector fields $y_{A}$ and $z^{A}$ are invariant, viz. $y_{A} c^{A}, z^{A} c_{A}=0$ and $\mathcal{L}_{c} y^{A}=0, \mathcal{L}_{c} z^{A}=0$, and they obviously project down to $y_{i}$ and $w^{i}$, i.e. $\mathcal{C}_{*}^{\downarrow}\left(y_{A}\right)=y_{i}$ and $\mathcal{C}_{*}^{\downarrow}\left(z^{A}\right)=w^{i}$. The assertion now follows since $\mathcal{C}_{*}^{\uparrow}$ is an isomorphism.

Remark. The preceding exposition is consistent with the fibre bundle formulation of gauge theory [2]. In the general setting, the fibres are not necessarily orbits of isometries. This entails, in particular, that there is a priori no natural "lift" of a vector field, but any connection on the bundle specifies a "horizontal lift". In the present case, however, Killing transport provides a natural connection, and our lift is indeed "horizontal" in this sense. What we have done in (8) and (9) now reads, in gauge theoretic language, to decompose the horizontal lifts of vector fields in terms of a "direct product basis" (5). Applied to the basis vectors themselves, this corresponds to the introduction of a "gauge covariant derivative" ( cf Sect. 5 of [2]). 


\section{Horizons}

\subsection{Killing horizons}

We recall here a known result (cf. e.g. Sect. 4 of [6])

Theorem 1. In an Einstein-Maxwell-dilaton spacetime $\left(\mathcal{M}, g_{i j}\right)$ let $\mathcal{H}$ be a Killing horizon with generator $l^{i}$ and surface gravity $\kappa$. That is to say, $\mathcal{H}$ is an embedded $3 \mathrm{~d}$ null surface and $l^{i}$ is a Killing field defined in a neighbourhood of $\mathcal{H}$ and future directed and null on $\mathcal{H}$ where it satisfies $l^{i} \nabla_{i} l^{j}=\kappa l^{j}$. Assume furthermore that $\mathcal{L}_{l} A_{i}=0$ and $\mathcal{L}_{l} v=0$. Then the lift ${ }^{5} \mathcal{H}=\mathcal{C}^{\uparrow}(\mathcal{H})$ is a Killing horizon in the $5 \mathrm{~d}$ vacuum spacetime $\left({ }^{5} \mathcal{M},{ }^{5} g_{A B}\right)$ with generator $h^{A}=l^{A}-\varphi c^{A}$ where $l^{A}=\left(l^{i}, 0\right)$ and $\varphi=\left.2 A_{i} l^{i}\right|_{\mathcal{H}}$. Moreover, the surface gravity ${ }^{5} \kappa$ defined via $h^{A}{ }^{5} \nabla_{A} h^{B}={ }^{5} \kappa h^{B}$ on ${ }^{5} \mathcal{H}$ agrees with $\kappa$. Finally, on ${ }^{5} \mathcal{H}, h^{A}$ coincides with the lift $\mathcal{C}_{*}^{\uparrow}\left(l^{i}\right)=l^{A}-\Phi c^{A}$ where $\Phi=\mathcal{C}_{*}^{\uparrow}(\phi)$ and $\phi=2 A_{i} l^{i}$.

We recall that $\kappa$ and $\varphi$ are constant on the horizon [31]; However, these constants are not well defined as $l^{i}$ is only fixed up to a multiplicative constant, and since, moreover, there is the gauge freedom $A_{i}^{\prime}=A_{i}+\nabla_{i} \Lambda$. However, as also well known, there is a natural way of fixing these constants in the asymptotically flat setting. Such a fixing is not necessary in the present context.

Proof of Theorem 1. We first note that $h^{A}$ is null on ${ }^{5} \mathcal{H}$ :

$$
\left.h^{A} h_{A}\right|_{\mathcal{H}}=\left[v^{2}\left(\varphi-2 A_{i} l^{i}\right)^{2}+v^{-1} g_{i j} l^{i} l^{j}\right]_{\mathcal{H}}=0 \text {. }
$$

Next, $l^{A}$ is a Killing vector as $l^{i}$ leaves invariant all components of ${ }^{5} g_{A B}$ (cf (5) ). Since $h^{A}$ is a constant linear combination of two Killings, it is Killing as well. The remaining statements of the theorem now follow from

$$
\begin{aligned}
h^{A}{ }^{5} \nabla_{A} h_{B} & =-\frac{1}{2}{ }^{5} \nabla_{B}\left(h^{A} h_{A}\right)=-\frac{1}{2} \mathcal{C}_{*}^{\uparrow}\left(\nabla_{i}\left(v^{-1} l^{j} l_{j}\right)\right)= \\
& =\mathcal{C}_{*}^{\uparrow}\left(v^{-1} l^{j} \nabla_{j} l_{i}\right)=\kappa \mathcal{C}_{*}^{\uparrow}\left(v^{-1} l_{i}\right)=\kappa h_{B}
\end{aligned}
$$

on ${ }^{5} \mathcal{H}=\mathcal{C}^{\uparrow}(\mathcal{H})$, where we have used adapted coordinates (5) and Eq. (10) .

Remark. Using the formalism developed and exposed in [34, 35, 36, 37, it should be possible to generalize this Theorem to cover isolated/non-expanding/non-evolving horizons for which the null vectors $l^{i}$ and $h^{A}$ are only Killing on $\mathcal{H}$ and ${ }^{5} \mathcal{H}$ respectively, but not necessarily in a neighbourhood. We do not go into details here.

\subsection{The expansion}

We now consider an orientable spacelike 2-surface $\mathcal{S} \subset \mathcal{M}$. As all subsequent considerations

will be local near $\mathcal{S}$, we can in fact restrict $\mathcal{M}$ to a neighbourhood of $\mathcal{S}$. We introduce future 
directed null vectors $l^{i}$ and $k^{i}$, not necessarily Killing but orthogonal to $\mathcal{S}$ and scaled such that $l^{i} k_{i}=-2$. These vectors can be extended off $\mathcal{S}$ by considering the null geodesics with these tangents. This allows to define the expansion of $l^{i}$ (or of the corresponding null geodesics) on $\mathcal{S}$ via

$$
\Theta=\left(g^{i j}+l^{(i} k^{j)}\right) \nabla_{i} l_{j}
$$

A standard result (cf e.g. Theorem 3.6.1 of [32]) reads that $\Theta$ is in fact independent of the way how $l^{i}$ has been extended.

An alternative way of writing the expansion is the following. Let $\mathcal{S}_{\lambda}$ be a family of orientable 2 -surfaces in a neighbourhood of $\mathcal{S}$, and let $\xi^{i}$ be the "lapse" of the foliation, i.e. the normal component of the corresponding flow vector. Moreover, let $\eta_{\lambda}$ be the volume form of $\mathcal{S}_{\lambda}$ and $H^{i}$ the mean curvature vector [32, 33] of $\mathcal{S}$. Then (cf Sect. 1 of [33])

$$
\mathcal{L}_{\xi} \eta_{\lambda}=\xi_{i} H^{i} \eta_{\lambda}
$$

(This formula appears more often in integrated form as "variation of area".) When $\xi^{i}$ coincides with the null direction $l^{i}$, (13) reduces to

$$
\mathcal{L}_{l} \eta_{\lambda}=\Theta \eta_{\lambda}
$$

which will be used below.

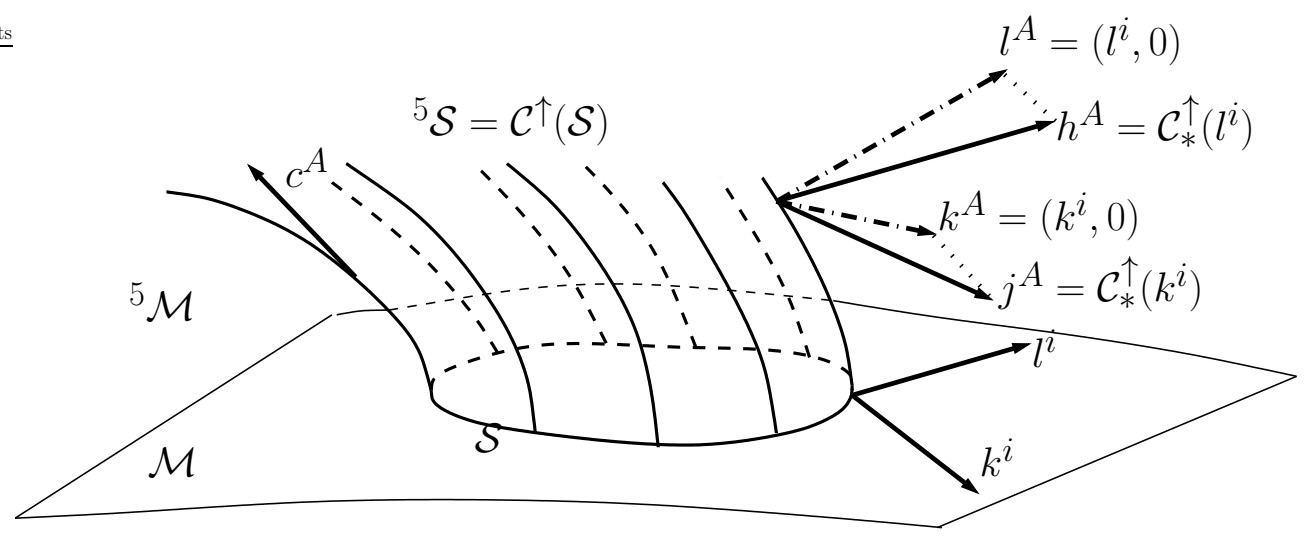

Figure 2: Lifts of null normals

For EMD theory ([6] $)$ we define $\psi=2 A_{i} k^{i}$ and we recall that $\phi=2 A_{i} l^{i}$. These quantities will in general not be constant on $\mathcal{S}$. We now lift the $\mathcal{S}_{\lambda}$ to 3 -surfaces ${ }^{5} \mathcal{S}_{\lambda}=\mathcal{C}^{\uparrow}\left(\mathcal{S}_{\lambda}\right)$, define $\Phi=\mathcal{C}_{*}^{\uparrow}(\phi)$ and $\Psi=\mathcal{C}_{*}^{\uparrow}(\psi)$, and lift $l^{i}$ and $k^{i}$ to invariant null vectors $\mathcal{C}_{*}^{\uparrow}\left(l^{i}\right)=h^{A}=l^{A}-\Phi c^{A}$ and $\mathcal{C}_{*}^{\uparrow}\left(k^{i}\right)=j^{A}=k^{A}-\Psi c^{A}$ on ${ }^{5} \mathcal{M}$ (cf Fig. 2). These vectors are orthogonal to ${ }^{5} \mathcal{S}$ since they are orthogonal to $c^{A}$, and since for all tangents $m^{i}$ to $\mathcal{S}$ and $m^{A}=\mathcal{C}_{*}^{\uparrow}\left(m^{i}\right)$ to ${ }^{5} \mathcal{S}$ we have $0=\mathcal{C}_{*}^{\uparrow}\left(m_{i} l^{i}\right)=m_{A} h^{A}$ and $0=\mathcal{C}_{*}^{\uparrow}\left(m_{i} k^{i}\right)=m_{A} j^{A}$. We also note that ${ }^{5} g_{A B} h^{A} j^{B}=-2 V^{-1}$, and in the same way as in (12) and (13) we define the expansions of $h^{A}$ :

$$
\begin{aligned}
{ }^{5} \Theta & =\left({ }^{5} g^{A B}+V h^{(A} j^{B)}\right){ }^{5} \nabla_{A} h_{B}, \\
\mathcal{L}_{h}{ }^{5} \eta_{\lambda} & ={ }^{5} \Theta{ }^{5} \eta_{\lambda},
\end{aligned}
$$


where ${ }^{5} \eta_{\lambda}$ is the volume element of ${ }^{5} \mathcal{S}$.

Alternatively, we could of course start from $3 \mathrm{~d}$ surfaces ${ }^{5} \mathcal{S}_{\lambda} \subset{ }^{5} \mathcal{M}$ which are invariant under an isometry. They project to $2 \mathrm{~d}$ surfaces $\mathcal{S}_{\lambda} \subset \mathcal{M}$, and the null normals and expansions are related as above.

We now have the following result which we prove in two ways.

Theorem 2. Let $\mathcal{S} \subset \mathcal{M}$ and ${ }^{5} \mathcal{S} \subset{ }^{5} \mathcal{M}$ be orientable spacelike surfaces such that $\mathcal{S}=$ $\mathcal{C}^{\downarrow}\left({ }^{5} \mathcal{S}\right)={ }^{5} \mathcal{S} / \mathcal{C}$ where $\mathcal{C}$ is an isometry. Let $l^{i}$ and $h^{A}=\mathcal{C}_{*}^{\uparrow}\left(l^{i}\right)$ be the respective null normals related by the Geroch-isomorphism. Then the corresponding null expansions defined by (12) or (14) and (15) or (16) coincide in the sense that $\mathcal{C}_{*}^{\downarrow}\left({ }^{5} \Theta(h)\right)=\Theta(l)$.

First proof. We manipulate (15) till it projects down to (12). For ease of notation, we suppress the $\mathcal{C}_{*}^{\downarrow}-$ notation in both subsequent proofs. However, we have to take care to avoid ill-defined expressions such as $\mathcal{C}_{*}^{\downarrow}\left({ }^{5} \nabla_{A} h_{B}\right)$, which is accomplished by appropriate algebraic decomposition of ${ }^{5} \nabla_{A} h_{B}$. Next, to obtain (20) we have used (4) while the final step which leads to (23) uses the behaviour of the $4 \mathrm{~d}$ covariant derivative under conformal rescalings of the metric,

$$
\begin{aligned}
{ }^{5} \Theta(h) & =\left({ }^{5} g^{A B}+V h^{(A} j^{B)}\right){ }^{5} \nabla_{A} h_{B}= \\
& =\left({ }^{5} g^{A B}+V h{ }^{(A} j^{B)}\right)\left(P_{A}^{C}+V^{-2} c_{A} c^{C}\right)\left(P_{B}^{D}+V^{-2} c_{B} c^{D}\right){ }^{5} \nabla_{C} h_{D}= \\
& =\left({ }^{5} g^{A B}+V h^{(A} j^{B)}\right) P^{C}{ }_{A} P^{D}{ }^{5} \nabla_{C} h_{D}+V^{-2} c^{A} c^{B}{ }^{5} \nabla_{A} h_{B}= \\
& =\left(\widetilde{g}^{i j}+v l^{(i} k^{j)}\right) \widetilde{\nabla}_{i}\left(V^{-1} l_{j}\right)-v^{-2} c^{A} h^{B}{ }^{5} \nabla_{A} c_{B}= \\
& =v\left(g^{i j}+l^{(i} k^{j)}\right) \widetilde{\nabla}_{i}\left(v^{-1} l_{j}\right)+v^{-2} c^{A} h^{B}{ }^{5} \nabla_{B} c_{A}= \\
& =\left(g^{i j}+l^{(i} k^{j)}\right) \widetilde{\nabla}_{i} l_{j}+v^{-1} l^{i} \nabla_{i} v= \\
& =\left(g^{i j}+l^{(i} k^{j)}\right) \nabla_{i} l_{j}-v^{-1} l^{i} \nabla_{i} v+v^{-1} l^{i} \nabla_{i} v=\Theta(l) .
\end{aligned}
$$

Second Proof. Here the goal is to project (16) into (14). We first note that the volume forms are related via

$$
\eta_{\lambda}=v \widetilde{\eta}_{\lambda}=v i_{n}{ }^{5} \eta_{\lambda}=i_{c}{ }^{5} \eta_{\lambda}
$$

where $\widetilde{\eta}_{\lambda}$ are the 2 -surface elements on $\mathcal{S}$ arising from the conformally rescaled ambient metric $\widetilde{g}_{i j}=v^{-1} g_{i j}$, and $i_{n}$ and $i_{c}$ are inner products w.r.t. the unit vector $n^{A}=V^{-1} c^{A}$ and w.r.t $c^{A}$, respectively. From the definitions (14), (16), from (24) and since $h^{A}$ commutes with $c^{A}$ it now follows indeed that

$$
\Theta \eta_{\lambda}=\mathcal{L}_{l} \eta_{\lambda}=\mathcal{L}_{l}\left(i_{c}{ }^{5} \eta_{\lambda}\right)=\mathcal{L}_{h}\left(i_{c}{ }^{5} \eta_{\lambda}\right)=i^{c} \mathcal{L}_{h}{ }^{5} \eta_{\lambda}={ }^{5} \Theta i^{c}{ }^{5} \eta_{\lambda}={ }^{5} \Theta \eta_{\lambda}
$$

Remark. The correct conformal scaling as in (5) is crucial both for the Kaluza-Klein decomposition (6) as well as for the previous result. Only in the special case where $l^{i}$ leaves $v$ 
invariant, i.e. $l^{i} \nabla_{i} v=0$, the conformal scaling is irrelevant.

The previous result suggests the following definition.

Definition 1. We define a MOTS $\mathcal{S} \subset \mathcal{M}$ to be a compact, connected, orientable and embedded 2-surface with vanishing null expansion, i.e. $\Theta(l)=0$. Analogously, a MOTS ${ }^{5} \mathcal{S} \subset{ }^{5} \mathcal{M}$ is a compact, connected, orientable and embedded 3-surface with ${ }^{5} \Theta(h)=0$.

We remark that there have also been considered non-orientable MOTS [18] and nonembedded MOTS [12]. The present restrictive definition is required for some results of Sect. 4 .

\subsection{Stability and symmetry of MOTS}

With the proper conformal scaling of the ambient metric, Theorem 2 shows that a MOTS $\mathcal{S} \subset \mathcal{M}$ lifts to a surface ${ }^{5} \mathcal{S}=\mathcal{C}^{\uparrow}(\mathcal{S}) \subset{ }^{5} \mathcal{M}$, with vanishing expansion. As $\mathcal{S}$ was required to be embedded, the same is true for ${ }^{5} \mathcal{S}$. The reason is that ${ }^{5} \mathcal{M}$ has locally a product structure near each point of ${ }^{5} \mathcal{S}$; so in particular any self intersection ${ }^{5} \mathcal{S}$ would project down to a self intersection on $\mathcal{S}$. It follows that ${ }^{5} \mathcal{S}$ is also a MOTS. On the other hand, essentially the same reasoning shows that an invariant $\operatorname{MOTS}{ }^{5} \mathcal{S} \subset{ }^{5} \mathcal{M}$ projects to a MOTS $\mathcal{S}=\mathcal{C}^{\downarrow}\left({ }^{5} \mathcal{S}\right)$.

In view of the significance of the property of stability as outlined in the introduction, we wish to show that it is preserved upon projections and lifts as well. Intuitively, this is clear because stability of an n-dim. MOTS means existence of an $n+1$ dim. neighbourhood whose interior and exterior parts can be foliated by outer trapped and outer untrapped surfaces, respectively, which means that the expansions of the leaves have appropriate signs. Such a foliation should then project and lift as well, and these manipulations should preserve the respective expansions due to Theorem 2. However, the details of this argument which lead to Theorem 3 involve subtleties. In particular, we will need two definitions of stability given in [11, 12]. We recall below, for 2d MOTS $\mathcal{S} \subset \mathcal{M}$, the definition which was called "(strictly) stably outermost" in [11, 12], and at the risk of boring the reader we explicitly state and discuss its rather trivial extension to $3 \mathrm{~d} \operatorname{MOTS}{ }^{5} \mathcal{S} \subset{ }^{5} \mathcal{M}$. The alternative definition (not repeated below) requires non-negativity (positivity) of the principal eigenvalue of a linear elliptic "stability operator". These definitions are equivalent, and this equivalence will play a role in Theorem 3 below.

\section{Definition 2.}

a.) Stability in 4d: A MOTS $\mathcal{S} \subset \mathcal{M}$ is called stable w.r.t. a normal direction $m^{i}$ iff there exists a variation $\delta_{\psi m}$ with $\psi \geq 0, \psi \not \equiv 0$ such that $\delta_{\psi m} \Theta \geq 0$. The MOTS is strictly stable iff it is stable with $\delta_{\psi m} \Theta \not \equiv 0$.

b.) Stability in 5d: A MOTS ${ }^{5} \mathcal{S} \subset{ }^{5} \mathcal{M}$ is called stable w.r.t. a normal direction $n^{A}$ iff there exists a variation $\delta_{\Psi n}$ with $\Psi \geq 0, \Psi \not \equiv 0$ such that $\delta_{\Psi n}{ }^{5} \Theta \geq 0$. The MOTS is strictly stable iff it is stable with $\delta_{\Psi n}{ }^{5} \Theta \not \equiv 0$. 
A priori a MOTS ${ }^{5} \mathcal{S} \subset{ }^{5} \mathcal{M}$ need not be invariant, nor need the direction of stability be invariant under the isometry. However, these invariance conditions will be key in the subsequent results, and in the next section the isometry group $U(1) \times U(1)$ will be considered. This motivates the following definition.

\section{Definition 3.}

a.) Stability w.r.t. an invariant direction in 4d: A MOTS $\mathcal{S} \subset \mathcal{M}$ which is invariant under an isometry $\mathcal{C}$ is called stable with respect to an invariant normal direction $m^{i}$ if Definition 2 a.) applies and if $\mathcal{L}_{c} m^{i}=0$.

b.) Stability w.r.t. an invariant direction in 5d: A MOTS ${ }^{5} \mathcal{S} \subset{ }^{5} \mathcal{M}$ which is invariant under an isometry group $G$ is called stable with respect to an invariant normal direction $\mathbf{n}^{A}$ if Definition 2 b.) applies and if $\mathcal{L}_{g} n^{A}=0$ for all elements $g^{A}$ of the Lie algebra $\mathcal{G}$ of $\mathrm{G}$.

These definitions apply to any normal directions $m^{i}$ or $n^{A}$. However, Theorem 3 below requires the outgoing conditions $m_{i} l^{i}>0$ or $n_{A} h^{A}>0$ (as it uses results of [12]); note that this condition still admits directions of any causal character. On the other hand, the area inequalities considered in Sect. 4 require in addition that $m^{i}$ and $n^{A}$ are achronal (spacelike or null). All subsequent results apply if $m^{i}$ and $n^{A}$ are achronal and outgoing. The limiting cases are $m^{i}=l^{i}$ or $n^{A}=h^{A}$ and $m^{i}=-k^{i}$ or $n^{A}=-j^{A}$. In the former case the variations considered above reduce to the Raychaudhuri equation and the stability conditions become highly restrictive, while the latter choices lead to the least restrictive conditions.

We remark that in Definition 3 the requirement that the MOTS itself is invariant could be relaxed once the stability condition is imposed. In fact there is the following relation between these two invariance properties: If the MOTS is stable in a normal direction $n^{A}$, then any Killing field $d^{A}$ on ${ }^{5} \mathcal{M}$ is either tangent to the MOTS or its normal component $d_{\perp}^{A}$ necessarily lies in the conical segment between the null generator $h^{A}$ of the MOTS and $n^{A}$ (cf. Theorem 8.1. of [12] and the subsequent discussion). Loosely speaking, a stable MOTS is automatically invariant under symmetries of the ambient space provided the stability direction cooperates. However, we do not exploit this fact in any of the subsequent results in order to keep their formulation simple.

\section{Theorem 3.}

1. Let $\mathcal{S} \subset \mathcal{M}$ be a MOTS which is (strictly) stable w.r.t. an outgoing normal direction $m^{i}$. Then the lifted MOTS $\mathcal{C}^{\uparrow}(\mathcal{S}) \subset{ }^{5} \mathcal{M}$ is (strictly) stable w.r.t. the outgoing normal direction $n^{A}=\mathcal{C}_{*}^{\uparrow}\left(m^{i}\right)$.

2. Let ${ }^{5} \mathcal{S} \subset{ }^{5} \mathcal{M}$ be an invariant MOTS which is (strictly) stable w.r.t. the invariant outgoing normal direction $n^{A}$. Then the projected MOTS $\mathcal{S}=\mathcal{C}^{\downarrow}\left({ }^{5} \mathcal{S}\right) \subset \mathcal{M}$ is (strictly) stable w.r.t. the normal direction $m^{i}=\mathcal{C}_{*}^{\downarrow}\left(n^{A}\right)$. 


\section{Proof.}

1. This is the easy part: Let $\psi$ be the function in the definition of stability of $\mathcal{S} \subset \mathcal{M}$ such that $\delta_{\psi m} \Theta \geq 0$. Then the lifts $\Psi=\mathcal{C}_{*}^{\uparrow}(\psi)$ and $n^{A}=\mathcal{C}_{*}^{\uparrow}\left(m^{i}\right)$ satisfy the requirements for stability on ${ }^{5} \mathcal{S} \subset{ }^{5} \mathcal{M}$, in particular $\delta_{\Psi n}{ }^{5} \Theta \geq 0$. More explicitly, in terms of a coordinate chart $x^{i}$ near $\mathcal{S}$, and an adapted coordinate $z$ such that $\psi m^{i} \partial / \partial x^{i}=\partial / \partial z$, we have $\delta_{\psi m} \Theta=\partial / \partial z \Theta \geq 0$. Lifting all coordinates to a neighbourhood of ${ }^{5} \mathcal{S}$ we obtain the result.

2. In spite of the invariance assumptions there is still a subtlety here: The function $\Psi$ which defines the variation $\delta_{\Psi n}{ }^{5} \Theta \geq 0$ in Definition 3.b.) of stability of ${ }^{5} \mathcal{S}$ need not be invariant under $\mathcal{C}$, in which case it does not project to $\mathcal{S} \subset \mathcal{M}$. Here we need the elliptic machinery developed in [11, 12]. We rewrite the variation $\delta_{\Psi n}{ }^{5} \Theta$ in terms of a linear elliptic operator ${ }^{5} L_{n}$ acting on $\Psi$ such that $\delta_{\Psi n}{ }^{5} \Theta={ }^{5} L_{n} \Psi$. Any linear elliptic operator has a real, positive "principal" eigenfunction $\Phi$ corresponding to the "principal" eigenvalue $\lambda$ (the eigenvalue with lowest real part), viz. ${ }^{5} L_{n} \Phi=\lambda \Phi$. For ${ }^{5} L_{n}$ introduced above, the definitions of stability and strict stability are equivalent to $\lambda \geq 0$ and $\lambda>0$, respectively. This means in particular that for a stable MOTS, the principal eigenfunction $\Phi$ defines a prefered class of variations such that $\delta_{\Phi n}{ }^{5} \Theta={ }^{5} L_{n} \Phi=\lambda \Phi \geq 0$. (or $>0$ in the strictly stable case). But by virtue of the invariance of the stability direction $n^{A}$, the stability operator commutes with the isometry, viz. $\mathcal{L}_{c}{ }^{5} L_{n}={ }^{5} L_{n} \mathcal{L}_{c}$. Now Theorem 8.2. of [12] implies that the principal eigenfunction is invariant under the isometry, i.e. $\mathcal{L}_{c} \Phi=0$. Hence $\Phi$ can be projected to $\mathcal{M}$ and $\phi=\mathcal{C}_{*}^{\downarrow} \Phi$ defines the variations on $\mathcal{S}$ which imply stability, in particular $\delta_{\phi m} \Theta \geq 0$.

\section{Area inequalities}

Area inequalities bound the area $A$ of stable MOTS in terms of quantities defined on the MOTS, namely charges and, in the axially symmetric case, angular momenta ([22, 23]). Here we first briefly recall the area inequalities for Einstein-Maxwell and Einstein-Maxwell-dilaton in $4 \mathrm{~d}$, and in a $5 \mathrm{~d}$ vacuum.

For axially symmetric, stable 2d MOTS in Einstein-Maxwell theory, Gabach Clement et al. [24, 25] proved that

$$
A \geq 4 \pi \sqrt{4 J^{2}+\left(Q^{2}+P^{2}\right)^{2}},
$$

where $J$ is the angular momentum and $Q$ and $P$ are the electric and magnetic charges. Equality holds iff the near horizon geometry is an extreme Kerr-Newman one.

In EMD theory, for couplings which include Einstein-Maxwell (i.e. no dilaton) as well as the Lagrangian (6) , Yazadjiev has shown (Theorems 1 and 2 of [26]) that for 2d MOTS which 
are stable w.r.t. an achronal, outgoing normal direction,

$$
A \geq 8 \pi \sqrt{\left|J^{2}-Q^{2} P^{2}\right|} .
$$

Moreover, for the KK coupling equality holds iff the (near-) horizon geometry [39, 40] is extreme and stationary.

On the other hand, in a $5 \mathrm{~d}$ vacuum spacetime with isometry group $U(1)^{2}=U(1) \times U(1)$ Hollands has obtained an area inequality for invariant $3 \mathrm{~d} \operatorname{MOTS}{ }^{5} \mathcal{S}$ which are stable w.r.t. an achronal, outgoing, invariant normal direction (Theorem 1 of [27]). It takes the form

$$
{ }^{5} A \geq 8 \pi \sqrt{\left|{ }^{5} J_{+}{ }^{5} J_{-}\right|}
$$

where ${ }^{5} J_{+}$and ${ }^{5} J_{-}$are angular momenta associated with the isometries. Again equality holds precisely for the stationary, extreme (near-) horizon geometries.

We now compare these inequalities. Regarding the relation between (26) and (27) the situation is clear: As already noticed in [26], the former is the stronger inequality but it only applies in the Einstein-Maxwell case. On the other hand, (27) and (28) should have a common range of applicability: As we have discused at length, a 5d vacuum with a spacelike isometry is equivalent to EMD theory in $4 \mathrm{~d}$, and the isometry preserves stable MOTS in the sense of Theorems 2 and 3.

However, regarding invariance, stability and topology the setup of [26] and [27] is different and in fact also different from our favourite setup as described in Sect. 3.3. We have to clarify these issues before investigating the relation between the parameters.

Invariance of the ambient space. Eqs. (27) and (28) hold, respectively, for MOTS which are invariant under the $U(1)$ and $U(1)^{2}$ symmmetry. However, invariance of the full ambient geometry is assumed in [27] but not in [24, 25, 26]. To keep the following discussion simple we always assume below symmetry of the full ambient geometry.

Invariance of the MOTS. In [27] where $U(1)^{2}$ invariance of the ambient geometry is assumed, it is stated that the considered MOTS ${ }^{5} \mathcal{S}$ is then automatically invariant under this symmetry. This is due to the very construction of that MOTS via an appropriate cross section of an invariant light cone. Here we assume, in contrast, that a MOTS ${ }^{5} \mathcal{S}$ is given, and accordingly we require henceforth its invariance under the ambient symmetry group. (See, however, the remark after Definition 3 regarding a possible relaxation of this requirement for stable MOTS).

Invariance of the stability direction. The proofs [24]-[27] of the area inequalities (26) - (28) agree more or less regarding the assumptions of compatibility of the stability direction with the symmetries. All these assumptions are formulated in terms of scalar functions with respect to suitably scaled null bases. However, in view of the discussion of the previous section, we prefer to set out from our purely geometric Definition 3 instead. Then the proof of the second part of Theorem 3 shows that there exist invariant lapse functions $\phi$ and $\Phi$. This implies in particular stability in the sense used in the proofs of the area inequalities. 
Topology of the MOTS. The MOTS ${ }^{5} \mathcal{S}$ are 3 -dimensional, connected, orientable, compact Riemannian manifolds with a two-dimensional isometry group containing $U(1)^{2}$. It has been shown in Theorem 2 of [41] that such surfaces are topologically one of $\mathbb{S}^{3}, \mathbb{S}^{2} \times \mathbb{T}$, the lens spaces $L(p, q)$, or $\mathbb{T}^{3}$ (where $p, q \in \mathbb{Z}$ with g.c.d. $(p, q)=1$, and $\mathbb{T}$ denotes a closed curve). The $\mathbb{T}^{3}$ topology is in fact incompatible with the stability condition [18] and will therefore not be considered further. On the other hand, (27) holds for MOTS of topology $\mathbb{S}^{2}$. Therefore, a necessary prerequisite for obtaining (27) from (28) upon dimensional reduction is that we set out from a MOTS ${ }^{5} \mathcal{S}$ which is a $\mathbb{T}$ bundle over $\mathbb{S}^{2}$. Such bundles have been classified [42] and they clearly include $\mathbb{S}^{3}$ and $\mathbb{S}^{2} \times \mathbb{T}$. As to the Lens spaces $L(p, q)$ for $p \geq 2, q \geq 2$, they involve discrete identifications in two directions. While one of these directions can be aligned with the $\mathbb{T}$ - fibre, the other one would lead to "orbifold-" identifications on $\mathbb{S}^{2}$ which we do not consider here. For the Lens spaces $L(p, 1)$, however, only the fibre identification remains, whence this case is admitted below as well. For the $\mathbb{S}^{2}$ bundles, Theorem 2 of [41] also shows that there is a "Kaluza-Klein-" subgroup $U(1) \subset U(1)^{2}$ which acts freely on ${ }^{5} \mathcal{S}$ and is aligned with the $\mathbb{T}$ - fibres.

Note that (28) holds for all lens spaces $L(p, q)$, not necessarily sphere bundles. Hence this inequality is more general than (27) in this sense.

We proceed with two Theorems. Theorem 4 just reviews key elements of the lift and projection procedures which are in particular needed to relate the requirements used in the proofs of (27) and (28). Its proof follows readily from Theorems 2 and 3 and the preceding remarks. In Theorem 5 we then define and analyze the parameters occuring in the area inequalities.

\section{Theorem 4.}

1. Let $\left(\mathcal{M}, g_{i j}, A_{i}, v\right)$ be an axially symmetric EMD spacetime containing a 2-dim MOTS $\mathcal{S}$ with "axial" isometry $\mathcal{A}$ (two fixed points) and corresponding Killing vector $\xi^{i}$. Then lifting to a 5 -dim vacuum spacetime $\left({ }^{5} \mathcal{M},{ }^{5} g_{A B}\right)$ with periodic isometry $\mathcal{C}$ and corresponding Killing vector $c^{A}$ gives an $\mathcal{A} \times \mathcal{C}$ invariant MOTS ${ }^{5} \mathcal{S}$ with additional symmetry $\Xi^{A}=\mathcal{C}_{*}^{\uparrow}\left(\xi^{i}\right)$. If $\mathcal{S}$ is stable w.r.t. an outgoing $\mathcal{A}$-invariant normal direction $m^{i}$ in the sense of Definition 3, then ${ }^{5} \mathcal{S}$ is stable w.r.t. to the $\mathcal{A} \times \mathcal{C}$-invariant direction $\mathcal{C}_{*}^{\uparrow}\left(m^{i}\right)$.

2. Conversely, let $\left({ }^{5} \mathcal{M},{ }^{5} g_{A B}\right)$ be a 5 -dim vacuum spacetime with a $U(1)^{2}$ isometry. Let ${ }^{5} \mathcal{S} \subset{ }^{5} \mathcal{M}$ be a MOTS which is topologically a $\mathbb{T}$ bundle over $\mathbb{S}^{2}$ (hence either $\mathbb{S}^{3}, \mathbb{S}^{2} \times \mathbb{T}$ or $L(p, 1))$ and which is invariant under the ambient isometries. Then there exist two $U(1)$ subgroups $\mathcal{C}$ and $\mathcal{A}$, the former acting freely on ${ }^{5} \mathcal{M}$, with corresponding Killing fields $c^{A}$ and $\Xi^{A}$. Dimensional reduction then yields an axially symmetric EMD spacetime $\left(\mathcal{M}, g_{i j}, A_{i}, v\right)$ containing a 2 -dim, axially symmetric MOTS $\mathcal{S}$ with axial Killing vector $\xi^{i}=\mathcal{C}_{*}^{\downarrow}\left(\Xi^{A}\right)$. Moreover, if ${ }^{5} \mathcal{S}$ is stable in an outgoing direction $m^{A}$ which is invariant under $U(1) \times U(1), \mathcal{S}$ is stable in a direction $\mathcal{C}_{*}^{\downarrow}\left(m^{A}\right)$ which is invariant under the axial isometry. 
Theorem 5. Let the spacelike surface ${ }^{5} \mathcal{S} \subset{ }^{5} \mathcal{M}$ be a bundle of topology $\mathbb{S}^{3}, L(p, 1)$ or $\mathbb{S}^{2} \times \mathbb{T}$, such that the base $\mathcal{S}=\mathcal{C}^{\downarrow}\left({ }^{5} \mathcal{S}\right)={ }^{5} \mathcal{S} / \mathcal{C}$ has topology $\mathbb{S}^{2}$ and the fibres $\mathcal{C}$ are isometries. Then the areas of ${ }^{5} \mathcal{S}$ and $\mathcal{S}$ ( w.r.t. the measures ${ }^{5} \eta$ and $\eta$ ) are related by ${ }^{5} A=Z A$ (where $Z$ is the periodicity of $x^{4}$ ), and charges $Q$ and $P$ are defined and related via (29) and (30)

$$
\begin{aligned}
Q & =\frac{1}{4 \pi} \int_{\mathcal{S}} v^{3} F_{i j} d S^{i j}=\frac{1}{4 \pi Z} \int_{{ }^{5}}{ }^{5} \nabla_{A} c_{B} d S^{A B} \\
P & =\frac{1}{4 \pi} \int_{\mathcal{S}} * F_{i j} d S^{i j}=\frac{1}{8 \pi Z} \int_{{ }^{5} \mathcal{S}} \epsilon^{A B C}{ }_{D E} V^{-2} c_{A}{ }^{5} \nabla_{B}\left(V^{-2} c_{C}\right) d S^{D E}
\end{aligned}
$$

Moreover, $8 \pi P=Z p$ where $p=0$ for topology $\mathbb{S}^{2} \times \mathbb{T}, p=1$ for $\mathbb{S}^{3}$ and $p$ agrees with the lens space parameter otherwise.

Furthermore, if $\mathcal{S} \subset \mathcal{M}$ and ${ }^{5} \mathcal{S} \subset{ }^{5} \mathcal{M}$ are axially symmetric, their angular momenta are

$$
J=\frac{1}{8 \pi} \int_{\mathcal{S}} \nabla_{i} \xi_{j} d S^{i j}=\frac{1}{8 \pi Z} \int_{5_{\mathcal{S}}} \nabla_{A} \Xi_{B} d S^{A B} .
$$

Proof. The area $A$ of any invariant 2 -surface $\mathcal{S}$ is related to the area ${ }^{5} A$ of its lift ${ }^{5} \mathcal{S}=\mathcal{C}_{*}^{\uparrow}(\mathcal{S})$ via

$$
{ }^{5} A=\int_{{ }^{5} \mathcal{S}}{ }^{5} \eta=\int_{{ }^{5} \mathcal{S}} \tilde{\eta} \wedge n=\int_{0}^{Z} \int_{\mathcal{S}} \eta \wedge d x^{4}=Z A,
$$

where $\widetilde{\eta}$ refers to the metric $\widetilde{g}_{i j}$, and $n=V d x^{4}$ is the 1 -form dual to the unit vector $n^{A}=V^{-1} c^{A}$.

To show the equivalence of the two representations in (29), (30) and (31) we first note that the surface element on ${ }^{5} \mathcal{S}$ reads $d S^{A B}=V h^{[A} j^{B]}{ }^{5} \eta$ in terms of the lifts $h^{A}=\mathcal{C}_{*}^{\uparrow}\left(l^{i}\right)$ and $j^{A}=\mathcal{C}_{*}^{\uparrow}\left(k^{i}\right)$, as the latter are not normalized but scale as $h^{A} j_{A}=-2 V^{-1}$, cf Sect. 3.2.

Recalling now (7) we find that the integrands are related as follows

$$
\begin{aligned}
\mathcal{C}_{*}^{\downarrow}\left[\left({ }^{5} \nabla_{A} c_{B}\right) h^{A} j^{B}\right] & =\mathcal{C}_{*}^{\downarrow}\left[V^{2}{ }^{5} \nabla_{[A}\left(V^{-2} c_{B]}\right)\right] \mathcal{C}_{*}^{\downarrow}\left(h^{A} j^{B}\right)= \\
& =2 v^{2}\left(\nabla_{[i} A_{j]}\right) l^{i} k^{j}=v^{2} F_{i j} l^{i} k^{j}, \\
\mathcal{C}_{*}^{\downarrow}\left[\epsilon_{D E}^{A B C}{ }_{D E} V^{-2} c_{A}{ }^{5} \nabla_{B}\left(V^{-2} c_{C}\right) h^{D} j^{E}\right] & =v^{-1} \epsilon_{i j}{ }^{k l} F_{k l} k^{i} l^{j}, \\
\mathcal{C}_{*}^{\downarrow}\left[\left(\nabla_{A} \Xi_{B}\right) h^{A} j^{B}\right] & =v^{-1} \nabla_{i} \xi_{j} k^{i} l^{j} .
\end{aligned}
$$

Finally, to show the relation $8 \pi P=Z p$ we recall a calculation in gauge theory which appears frequently in spherically symmetric settings (cf. e.g. Example 10.1 and Sect. 10.5.2 of [43]) but which we perform here in general. We consider domains $\mathcal{U}$ and $\mathcal{D}$ such that $\mathcal{U} \cup \mathcal{D}=\mathcal{S}$ and a smooth closed path $\mathcal{P} \subset \mathcal{U} \cap \mathcal{D}$ with unit tangent $t^{i}$ and parameter length $2 \pi$. On either domain we have connection 1 -forms $A_{i}^{U}$ and $A_{i}^{D}$ related by a gauge transformation $\Lambda: \mathcal{U} \cap \mathcal{D} \rightarrow U(1)$ with $A_{i}^{U}-A_{i}^{D}=\nabla_{i} \Lambda$. Upon integration, we obtain

$$
\begin{aligned}
8 \pi P & =2 \int_{\mathcal{S}} * F_{i j} d S^{i j}=2 \int_{\mathcal{S}} \epsilon_{i j k l} \nabla^{i} A^{j} d S^{k l}= \\
& =2 \int_{\mathcal{P}} A_{i}^{U} t^{i} d S-2 \int_{\mathcal{P}} A_{i}^{D} t^{i} d S=2 \int_{\mathcal{P}} \nabla_{i} \Lambda t^{i} d S=2[\Lambda(2 \pi)-\Lambda(0)] .
\end{aligned}
$$


Thus, when $P \neq 0, \Lambda$ is multi-valued on $\mathcal{U} \cap \mathcal{D}$. We now recall from Sect. 2 that the gauge transformation $A_{i} \rightarrow A_{i}+\nabla_{i} \Lambda$ corresponds to the motion $x^{4} \rightarrow x^{4}+2 \Lambda$ in the $U(1)$ fibre. Definiteness of the connection requires that $\Lambda(\mathcal{P})$ defines a closed orbit on the torus $\mathcal{P} \times U(1)$, so that moving once around $\mathcal{P}$ corresponds to $p$ loops around the fibre. In other words, there is a homotopy from $\mathcal{P}$ to $U(1)$ with winding number $p$. With a fibre of length $Z$, this gives $8 \pi P=2[\Lambda(2 \pi)-\Lambda(0)]=Z p$ as claimed. We remark that the monopole $P$ also agrees with the first Chern number of the bundle (cf. Example 11.2 of [43]).

Remark. The second representation (30) of the magnetic monopole provides an interesting connection with the Hopf invariant [43, 44] of the bundle. To see this recall that

$$
\frac{1}{8 \pi P} F_{i j}=\frac{1}{4 \pi P} \nabla_{[i} A_{j]}
$$

is a generator of the second cohomology group $H^{2}(\mathcal{S})$ which is non-trivial for $\mathbb{S}^{2}$. Hence (38) holds in general only locally. On the other hand, if ${ }^{5} \mathcal{S}$ has topology $\mathbb{S}^{3}$ or lens space topology $L(p, 1)$, the lift is exact globally, viz.

$$
\mathcal{C}_{*}^{\uparrow}\left[\frac{1}{8 \pi P} F_{k l}\right]=\left[{ }^{5} \nabla_{[A}\left(\frac{c_{B]}}{8 \pi P V^{2}}\right)\right] .
$$

In either case, the isometry $\mathcal{C}$ provides a map $\mathcal{C}^{\downarrow}: \mathbb{S}^{3} \rightarrow \mathbb{S}^{2}$ with Hopf invariant

$$
H(\mathcal{C})=\int_{5 \widehat{\mathcal{S}}} \epsilon^{A B C}{ }_{D E} \frac{c_{A}}{8 \pi P V^{2}}{ }^{5} \nabla_{B}\left(\frac{c_{C}}{8 \pi P V^{2}}\right) d S^{D E}
$$

where ${ }^{5} \widehat{\mathcal{S}}$ coincides with $\mathcal{S}$ for $\mathbb{S}^{3}$ topology but is the p-fold $\mathbb{S}^{3}$-cover in the case of the lens space $L(p, 1)$.

We now combine this with (30), take into account that the integral over ${ }^{5} \widehat{\mathcal{S}}$ is $p$ times the integral over ${ }^{5} \mathcal{S}$ and use $8 \pi P=Z p$. It follows that $H(\mathcal{C})=1$; in particular the Hopf invariant (which is an integer for any smooth map $\mathbb{S}^{3} \rightarrow \mathbb{S}^{2}$ ) does not reflect the winding number $p$.

We now define Killing vectors

$$
\Upsilon_{ \pm}^{A}= \pm 2 P c^{A}+\Xi^{A}= \pm \frac{Z p}{4 \pi} c^{A}+\Xi^{A}
$$

and corresponding Komar integrals

$$
{ }^{5} J_{ \pm}=\frac{1}{8 \pi} \int_{{ }^{5} \mathcal{S}}{ }^{5} \nabla_{A} \Upsilon_{B}^{ \pm} d S^{A B}
$$

which from the definitions (29)-(31) gives the further relation ${ }^{5} J_{ \pm}=Z(J \pm P Q)$.

To complete the identification of Theorems 1 of [26] and [27] containing (27) and (28) respectively, requires two trivial inputs. Firstly, in [27] all Killing vectors have period $2 \pi$, while our isometry $\mathcal{C}$ has a (dimensional) length $Z$. This can of course be fixed by adjusting 
units. Secondly, the integer $p$ defined in Theorem 5 matches the synonymous integer defined above Theorem 1 of [27], while we have to set the integer $q$ of [27] equal to 1 for the topological reason discussed above Theorem 4.

As already mentioned the extreme and stationary geometries in which (27) and (28) are saturated are included in the "near horizon geometries" analysed in [39, 40]. Among them, there is a large class of solutions which are known globally [4]: For the trivial bundle $p=0$ (topology $\left.\mathbb{S}^{2} \times \mathbb{T}\right)$, these include the boosted extremal Kerr and the black ring topologies, while for $p=1$ (topology $\mathbb{S}^{3}$ ) the geometries are either the extremal Myers-Perry or the extremal rotating Kaluza-Klein ones. For these stationary solutions, the relation between the $4 \mathrm{~d}$ and 5d parametes has been obtained and discussed in [38] (cf Appendix A2 in particular; again units need to be adjusted).

In this context we finally note that, from Theorem 1 , the property of extremality $(\kappa=0)$ of a stationary Killing horizon is preserved upon lift and projection, irrespective of any additional symmetries. This behaviour is of course reflected in the axially symmetric examples mentioned above. Moreover, it should extend to isolated/non-expaning/non-evolving horizons by virtue of the results of [37, 45].

Acknowledgements. We are grateful to Piotr Bizoń, Andreas Čap, Piotr Chruściel, Jose-Luis Jaramillo, Marc Mars and Helmuth Urbantke for helpful discussions and correspondence. We also (or rather: in particular) thank the referees for important comments which

led to substantial improvements. The research of W.S. was funded by the Austrian Science Fund (FWF): P 23337-N16

\section{References}

[1] Overduin J M and Wesson P S 1997 Phys. Rep. 283303

[2] Cho Y M 1975 J. Math. Phys. 162029

[3] Emparan R and Reall H S 2008 Living Rev. Rel. 11:6

[4] Horowitz G T and Wiseman T in 2012 Horowitz G T (ed.) Black Holes in Higher Dimensions (Cambridge: Cambridge University Press)

[5] Gibbons G 1982 Nucl. Phys. B 207337

[6] Simon W 1985 Gen. Rel. Grav. 17439

[7] Rasheed D 1995 Nucl. Phys. B 454379

[8] Larsen F 2000 Nucl. Phys. B 575211

[9] Hollands S and Ishibashi A 2012 Class. Quantum Grav. 29163001

[10] Jaramillo J L 2011 Int. J. of Modern Physics D 202169 
[11] Andersson L, Mars M and Simon W 2005 Phys. Rev. Lett. 95111102

[12] Andersson L, Mars M and Simon W 2008 Adv. Theor. Math. Phys 12853

[13] Andersson L and Metzger J 2009 Commun. Math. Phys 290941

[14] Eichmair M 2009 J. Diff. Geom 83551

[15] Andersson L, Eichmair M and Metzger J in 2009 Agranovsky M et al. (eds) Proceedings of the Fourth International Conference on Complex Analysis and Dynamical Systems (Contemporary Mathematics 553) (Providence: American Mathematical Society and RamatGan: Bar-Ilan University)

[16] Hawking S W and Ellis G F R 1973 The large scale structure of space-time (Cambridge: Cambridge University Press).

[17] Newman R P A C 1987 Class. Quantum Grav. 4277

[18] Galloway G in 2012 Horowitz G T (ed.) Black Holes in Higher Dimensions (Cambridge: Cambridge University Press)

[19] Andersson L, Mars M, Metzger J and Simon W 2009 Class. Quantum Grav. 26085018

[20] Costa e Silva I P 2012 Class. Quantum Grav. 29235008

[21] Jaramillo J L, Reiris M, Dain S 2011 Phys Rev. D 84 121503(R)

[22] Dain S 2012 Class. Quantum Grav. 29073001

[23] Jaramillo J L 2013 Springer Proceedings in Mathematics \&3 Statistics 26139

[24] Gabach Clement M E and Jaramillo J L 2012 Phys. Rev. D 86064021

[25] Gabach Clement M E, Jaramillo J L and Reiris M 2013 Class. Quantum Grav. (to be published)

[26] Yazadjiev S 2013 Phys. Rev. D 87024016

[27] Hollands S 2012 Class. Quantum Grav. 29065006

[28] Geroch R 1971 J. Math. Phys. 12, 918

[29] Pauli W in 1955 Jordan P Schwerkraft und Weltall (Braunschweig: F. Vieweg und Sohn)

[30] Cho Y M 1992 Phys. Rev. Lett. 683133

[31] Carter B in 1973 DeWitt C \& DeWitt B S (eds.) Black hole equilibrium states (New York: Gordon \& Breach) 
[32] Jost J 1998 Riemanian Geometry and Geometric Analysis (Berlin, Heidelberg, New York: Springer)

[33] Li P 2012 Geometric analysis (Cambridge: Cambridge University Press).

[34] Ashtekar A and Krishnan B 2004 Living Rev. Rel. 7:10

[35] Gourgoulhon E and Jaramillo J L 2006 Phys.Rept. 423159

[36] Booth I 2013 Phys.Rev. D 87024008

[37] Mars M 2012 Class. Quantum Grav. 29145019

[38] Emparan R and Maccarrone A 2007 Phys Rev. D 75084006

[39] Kunduri H K and Lucietti J 2009 J. Math. Phys. 50082502

[40] Hollands S and Ishibashi A 2010 Ann. Henri Poincaré 101537

[41] Hollands S and Yazadjiev S 2011 Commun. Math. Phys 302631

[42] Steenrod N 1951 The Topology of Fibre Bundles (Princeton: Princeton University Press)

[43] Nakahara M 2003 Geometry, Topology and Physics (Boca Raton: Taylor \& Francis Group)

[44] Bott R and Tu L W 1982 Differential Forms on Algebraic Topology (Berlin, Heidelberg, New York: Springer)

[45] Jaramillo J L 2012 Class. Quantum Grav. 29177001 